\title{
The Interpretation of Reversed Magnetization in Igneous Rocks
}

\author{
By J.H. PARRY \\ Department of Geodesy and Geophysics, Cambridge University
}

It has been established (Gelletich, Bruckshaw, Hospers etc.) that reversed magnetization is not a rare phenomenon but occurs in continuous series of lavaflows and groups of dykes alternating with other series and groups showing normal polarization.

Two hypotheses have been put forward to account for this. An external agency namely the reversal of the geomagnetic field and an internal effect due to various suggested types of magnetic interaction amongst the ferromagnetic constituents of the rock. I propose to discuss critical evidence for this internal self-reversal of permanent magnetization.

Néel has suggested 4 mechanisms giving this effect.

Néel I, an anti-ferromagnetic effect within the crystal lattice of the magnetic minerals such as has been found in the spinel $\mathrm{Li}_{0.5} \mathrm{Fe}_{1.25} \mathrm{Cr}_{1.25} \mathrm{O}_{4}$ (Gorter, Schulkes)

Néel II, a similar effect in which an original inferior magnetization of the inverse B lattice becomes predominant by subsequent demagnetization or destruction of the originally predominant A lattice.

Néel III, a magnetic interaction between two distinct substances, perhaps intergrown, with different Curie points and temperature coefficients of spontaneous magnetization, in which the second cools through its Curie point in the resultant of the geomagnetic field and the demagnetizing field of the first thus acquiring a reversed magnetization superior to the normal one of the first.

Néel IV, a similar mechanism in which the originally inferior reversed magnetization of the mineral of low Curie point only becomes dominant after the destruction or demagnetization of the other.

Graham has suggested and to some extent demonstrated the occurrence of a fifth mechanism. This consists of a subsequent alteration of a part of an originally single magnetic constituent to some other magnetic form, particularly oxidation of magnetite to maghemite. It is suggested that despite the low temperature the new material will be magnetized in the demagnetizing field of the original substance. The unaltered material is supposed to be demagnetized later owing to its larger grain size, leaving a reversed permanent magnetization due to the alteration product.

The rock found by Nagata at Mt. Haruna which definitely shows this property of self-reversal when heated and cooled in the laboratory appears to belong to type 
Néel III though certain experimental results obtained on it still require explanation.

The experimental methods so far used to investigate this problem are heating experiments, magnetic measurements at room temperature, mineralogical methods supported by physical studies such as X-ray photographs, and field studies:

Heating experiments are subject to the grave objection that alteration of the magnetic material is liable to occur but on the other hand such changes can be detected by the variation of properties other than those actually being measured in the experiments. It is perhaps most important to study only those rocks in which the thermo-remanent magnetization acquired in the present geomagnetic field does not differ in intensity by more than say $10 \%$ from the natural remanent magnetization in which case any very gross change can be eliminated. The decay of the natural remanent magnetization (N.R.M. after Nagata) with rise of temperature in zero field and the subsequent growth of thermo-remanent magnetization in the geomagnetic field as the temperature falls have been studied. Similarly the stepby-step destruction of N.R.M. and T.R.M. by heating to successively higher temperatures in zero field and cooling, again in zero field, to room temperature each time before measurment, has been carried out. Measurements of the curve of magnetization with temperature in fields of hundreds of gauss as carried out by Chevallier give an excellent value of the Curie point and are particularly sensitive to changes during heating. Given the variables of magnetic field, temperature and time many such experiments can be devised and in all cases changes such as oxidation which can be prevented must be by placing the specimen in a neutral atmosphere such as nitrogen or in a vacuum. The decay of N.R.M. and T.R.M. both continuously and partially seem significant as the rock was originally magnetized thermoremanently and they give a measure of the temperature at which this took place and the relation to the artificial effect from which changes may be detected.

Magnetic measurements in the cold can consist of determination of the complete hysteresis cycle or removal of the remanent magnetization by steady or alternating fields etc.. The initial susceptibility depends on the concentration of the magnetic material and the shape of the grains while the saturation intensity depends on the concentration and the nature of the material. Hence if one of these three factors is known the others may be deduced. The coercive force of the hysteresis cycle is related only to the nature, size, and shape of the material. If two minerals are present with different saturating fields a change of slope of the magnetization curve should occur when the first reaches saturation; such an effect has not been found with certainty in many cases. The destruction of the N.R.M. by a steady opposed field, the so-called coercive force of remanence, in relation to a similar destruction by an alternating field has been shown by Graham to be useful in detecting the presence of two magnetic materials of different "hardness."

Since the magnetic minerals form only a very small part of most rocks and the relation between their composition and that of the other minerals and of the rock as a whole is almost unknown, the standard mineralogical and petrological 
techniques of thin-section study and chemical analysis are not of great significance as yet. Polished section study is most useful in identifying the relationship between the ore-minerals, intergrowths and their development or disappearance on heating, oxidation products or the occurrence of irreversible changes such as that of maghemite to hematite. Complete and accurate separation of the ferromagnetic content from rocks so fine-grained as basalts is extremely difficult if not impossible; but even an imperfect separation can suffice to identify the minerals in question by X-ray methods, which is otherwise only possible by a Curie point determination where change may occur on heating.

Field studies show that reverse magnetization is not random, does not occur only in a particular petrological class of rocks and is related to similar reverse magnetization in sediments. Such considerations cannot be regarded as physical proof but they do point to a prima facie case for reversal of the earth's magnetic field requiring further investigation. Field work is needed to identify those rocks which are best to study where the magnetization, reversed or normal, is uniform within the rock and consistent from one flow to the next.

The minerals responsible for the magnetization of rocks and therefore those likely to be involved in the suggested modes of self-reversal are:

Magnetite and its solid solutions with Ulvöspinel, $\mathrm{Fe}_{2} \mathrm{TiO}_{4}$ giving the Titanomagnetites I ; with Ilmenite $\mathrm{FeTiO}_{3}$ giving the Titanomagnetites II (Chevallier); and probably with Maghemite (Hägg). The first two series have Curie points progressively lower than magnetite; data for the third are not available.

Maghemite $\mathrm{Fe}_{2} \mathrm{O}_{3} \gamma$ which is unstable and easily identified by its inversion on heating to haematite which is far less magnetic.

Haematite $\mathrm{Fe}_{2} \mathrm{O}_{3} \alpha$ which is only feebly ferromagnetic but may be of importance in igneous rocks, as it appears to be in sediments. Strongly ferromagnetic members of the solid solutions between haematite and ilmenite occur sparsely and may be of importance, that reported by Nagata in the Haruna pumice appears to be the first known occurrence in a lava,

The mineral sulphides of iron some of which are ferromagnetic are probably unimportant and are easily identified.

Impurities such as $\mathrm{Mg}, \mathrm{Na}, \mathrm{Al}$ etc. may modify the properties of most of the above but nearly always in the same sense and to a small extent in practice.

All these are anti-ferromagnetic.

With solid solutions, inversions, oxidations etc. many changes are possible in the normal history of a rock especially if there has been any degree of metamorphism, and during experiments, particularly heating.

Magnetite is liable to oxidation to maghemite or to haematite.

The titanomagnetites which with magnetite appear to be the most important in the basalts may exsolve into their end members; this has been shown to occur in slowly cooled rocks and may occur in time in basalts. There are indications of this effect in some Tertiary rocks, resolution occurring on heating. As in Graham's 
theory of self-reversal the magnetization of a material thus exsolved below its Curie point is important.

Maghemite is not an original mineral in igneous rocks but after its production by oxidation of magnetite it may invert to haematite.

Haematite is the end product of these changes and is not likely to alter except under very strongly reducing conditions.

It is necessary to draw conclusions from the hypotheses of self-reversal which can be tested in the laboratory or in the field.

Néel I If no change has occurred in time or in heating, the reversal would be observed on cooling from above the Curie point; if a change involving loss of the property occurs on heating the T.R.M. will probably be different from the N.R.M. and the saturation magnetization will change; a change in time would cause demagnetization of both sub-lattices since they are magnetically interdependent.

Néel II Dr. R. Street has recently pointed out to us that this mechanism is probably impossible as the two sub-lattices of an antiferromagnetic can only exist owing to the strong negative exchange interaction between them and therefore the destruction or demagnetization of one would involve that of the other. If a change took place it is unlikely that the new substance would be magnetized in the direction of the inferior B sub-lattice.

Néel III is the most interesting mechanism as it does not require the presence of any hitherto unknown mineral and has been shown to occur by Nagata; if neither mineral has been altered or demagnetized in time or by heating, the effect will be observed in the laboratory as in the Haruna pumice. If the normally magnetized mineral which has the higher Curie point has been demagnetized the temperature at which the N.R.M. is destroyed on heating in zero field ("Curie point of N.R.M.") will be lower in reversed than in normal rocks, the re-magnetization of the first will be observed on cooling, the T.R.M. will be of higher Curie point and a reversal may be observed. If the first mineral has been altered the Curie point of N.R.M. will again be low and the alteration product should be identifiable magnetically or otherwise; in both the above cases a difference between A.C. and D.C. demagnetization should occur. If a change occurs on heating the point at which it sets in is identifiable by the temperature at which the curve of magnetization in high fields against temperature (Chevallier) ceases to be reversible; it may occur above or below the Curie point. In all cases to prove the occurrence of the Néel III or IV mechanisms it is necessary to show that the mineral of higher Curie point is or was magnetized in the opposite direction to the other mineral. If the direction of magnetization cannot be demonstrated the question must be left open. On the other hand, if it can be shown that two minerals of different Curie points are magnetized in the same direction, these mechanisms can definitely be excluded. This is apparent from the continuous destruction of N.R.M. in zero field with increasing temperature in the case of the Iceland basalts.

The problem of the magnetization to be expected when an originally homo- 
geneous titanomagnetite exsolves at low temperature into two fractions of different Curie points or, as in Graham's hypothesis, when part of a finely intergrown magnetite is oxidised to maghemite, remains; Néel's treatment of the cooling of a mixture of two minerals is not strictly applicable since the first exsolved material will modify the field in which the exsolution will be completed whereas in the case of cooling all the material of lower Curie point is magnetized at one time and, the magnetization at that stage being small, the field is not modified. However if both materials are magnetized in the same direction the self-reversing mechanism is not applicable.

The self-reversal mechanisms so far proposed have been based largely on theoretical grounds. The arguments adduced against them and in support of reversals of the geomagnetic field have been mostly of an experimental and practical nature. The question will not be resolved until experiment and theory can meet and in particular until the properties of T.R.M. are better known and better explained.

\section{References}

Bruckshaw, J. McG. and Robertson E.I., Mon. Not. R. Astr. Soc., Geoph. Suppl., 5, 308-320 (1949).

Chevallier, R. and Pierre, J., Ann. de Physique, 18, 383 (1932).

Chevallier, R. and Girard, J., Bull. Soc. Chim. de France, 17, 576-81 (1950).

Gelletich, H., Beitr. Angew. Geoph., 6, 337-406 (1937).

Gorter, E.W., Philips Res. Rep., 9, 295-365, 403-44 (1954).

Graham, J.W., Journ. Geoph. Res., 58, 243-260 (1953).

Hägg, G., Zeits. Phys. Chem., 29B, 95 (1935).

Hospers, J., Kon. Ned. Ak. Wet., Proc., 56B, 467-491 (1953) ; Ibid., 57B, 112-121 (1954).

Nagata, T., Akimoto, S. and Uyeda, S., Proc. Jap. Acad., 27, $643-645$ (1951); Ibid., 28, 277-281 (1952).

Néel, L., Ann. de Géophys., 7, 90-102 (1951). 